\title{
Effects of complete replacement of fish oil with plant oil mixtures and algal meal on growth performance and fatty acid composition in juvenile yellowtail Seriola quinqueradiata
}

\author{
Haruhisa Fukada ${ }^{1} \mathbb{D} \cdot$ Renato Kitagima $^{2} \cdot$ Junpei Shinagawa $^{1} \cdot$ Haruka Morino ${ }^{1} \cdot$ Toshiro Masumoto $^{1}$
}

Received: 13 April 2019 / Accepted: 20 September 2019

(c) The Author(s) 2021, corrected publication 2021

\begin{abstract}
Docosahexaenoic acid (DHA) is an essential fatty acid for marine carnivorous fish. Algal meal (AM), available as a new dietary DHA source, could completely replace dietary fish oil (FO). In this study, dietary FO was replaced with plant oil mixtures and AM in juvenile yellowtail Seriola quinqueradiata to investigate its effects on growth performance and fatty acid composition. The FO control diet was prepared with only pollack liver oil as the lipid source. For the non-FO diets, pollack liver oil was completely replaced with mixtures of canola oil and palm oil, with AM supplementation at $0 \%$ (AM0), 1\% (AM1), 2\% (AM2), 3\% (AM3), and 4\% (AM4). After completion of the 8-week feeding trial, the AM2 group showed significantly higher values for final body weight and feed efficiency than the AM0 group. No significant differences were observed in the other parameters of growth performance. Whole-body fatty acid composition reflected the dietary fatty acid composition in all dietary groups. These findings demonstrate that AM is useful as a DHA source in yellowtail aquaculture, thus contributing to a reduction in the use of FO in fish diets.
\end{abstract}

Keywords Algal meal $\cdot$ Fatty acid $\cdot$ Fish oil replacement $\cdot$ Yellowtail

\section{Introduction}

Fish oil (FO) contains important essential fatty acids and is an energy source for aquaculture fishes. However, the production of FO is unstable and limited (Turchini et al. 2009; Hardy 2010), thus making it a restricting factor in aquaculture production. Sustainability and expansion of aquaculture requires the use of an alternative lipid source, such as various plant oils. The utilization of various alternative oils has been investigated in previous studies for many fishes (Turchini et al. 2009; Hardy 2010).

Marine carnivorous fish require n-3 long-chain polyunsaturated fatty acids (LC-PUFA), including docosahexaenoic acid (DHA) and eicosapentaenoic acid (EPA), as essential

Haruhisa Fukada

fukaharu@kochi-u.ac.jp

1 Faculty of Agriculture and Marine Science, Kochi University, 200 Monobe, Nankoku 783-8502, Kochi, Japan

2 Alltech Japan, 3-3-5 Tenjin, Chuo, Fukuoka 810-0001, Fukuoka, Japan nutrients. LC-PUFA are defined as those with carbon chain length $\geq 20$ and $\geq 2$ or 3 double bonds. FO is almost the only source of $n-3$ LC-PUFA in fish diets as this fatty acid is not present in plant oils (Sargent et al. 2002; Tocher 2010). Therefore, the complete replacement of FO in the diets of marine carnivorous fish, without any negative effects, has been difficult. However, a new DHA source, which is produced by marine microalgae, is now available.

Microalgae have high potential for oil production per area (Chisti 2007). Moreover, the price of plant oil has increased, similar to that of FO, and may further increase because of limited availability of arable land for food crops (Turchini et al. 2009). Furthermore, algal biomass produced by fermentation did not contain environmental contaminants and heavy metals (Ratledge 2004). In salmonids, partial FO replacement with a DHA-rich algal meal contributed to decreased organic pollutant levels in meat while maintaining almost the same level of DHA (Sprague et al. 2015). Considering this, microalgae are ideal candidates to ensure stable and sustainable production of a dietary oil source for fish feed, despite the high production costs (Turchini et al. 2009). Among 
microalgae, Aurantiochytrium sp. can produce oil and DHA at a high percentage (50-57\% dry matter and up to $49 \%$ fatty acid; Byreddy 2016; Sajjadi et al. 2018). Therefore, Aurantiochytrium sp. was utilized in studies on the replacement of FO in fish diets. Aurantiochytrium sp. oil was first used in the diet of the Atlantic salmon Salmo salar, and it was indicated as a good candidate for aquaculture feed (Miller et al. 2007). In a previous study of the Nile tilapia Oreochromis niloticus, lipid and fatty acids from dried Aurantiochytrium sp. showed good digestibility (Sarker et al. 2015). The utilization of Aurantiochytrium sp. as a dietary lipid was investigated in some fish species, and its availability as an alternative dietary lipid was confirmed for aquaculture fish. The complete replacement of FO using Aurantiochytrium sp. oil/meal has been undertaken in salmonids (Miller et al. 2007; Kousoulaki et al. 2015), tilapia (Sarker et al. 2016; Brignol et al. 2018), olive flounder Paralichthys olivaceus (Qiao et al. 2014), giant grouper Epinephelus lanceolatus (García-Ortega et al. 2016), and orange-spotted grouper E. coioides (Lee et al. 2017). The absence of a negative effect was also confirmed on the growth performance of longfin yellowtail fed with an algal meal (AM)-supplemented fish meal (FM)-based diet and FMreplaced diets (Kissinger 2016).

Yellowtail Seriola quinqueradiata is one of the most important aquaculture fish in Japan (Masumoto 2002) and requires relatively high concentrations of n-3 LC-PUFA (>2\%) (Deshimaru et al. 1982). Partial replacement (50\%) of dietary FO in juvenile yellowtail has been performed using palm oil and beef tallow; here, the growth performance was comparable to that of the control group (Aoki et al. 2000). In juvenile yellowtail, dietary FO was replaced with $0 \%, 25 \%$, $50 \%$, and $100 \%$ of canola oil; the $50 \%$ FO replacement group showed comparable growth to the control group (Fukada et al. 2017). In the present study, we aimed to investigate the utilization of commercial AM and the possibility of a non-FO diet for yellowtail.

\section{Materials and methods}

\section{Diets}

Six diets were prepared with identical dietary protein (49.0\%) and lipid (13.5\%) content (Table 1). Fish meal and krill meal were used as protein sources. Pollack liver oil, canola oil, palm oil, and AM were used as fat sources. Only pollack liver oil was used for the control diet (FO). Mixtures of canola oil and palm oil were used for the non-pollack liver oil diets. Plant oils were mixed so that a single fatty acid did not exceed $35 \%$ of fatty acid composition, because a large bias toward individual fatty acids might have been the cause for the low growth observed in previous studies (Fukada et al. 2017). A DHA-rich AM produced by Aurantiochytrium sp. (ALL-G RICH ${ }^{\mathrm{TM}}$, Alltech Inc., Lexington, KY, USA) was used as the DHA source. The quantities of the canola oil and palm oil mixtures were decreased with an increase in AM added at concentrations of $0 \%$ (AM0), 1\% (AM1), 2\% (AM2), 3\% (AM3), and 4\% (AM4). These were then thoroughly mixed in a mixer, and diets were pelletized $(3.2 \mathrm{~mm}$ in diameter) using a laboratory pellet mill (22VR-7500DX, Kire-Royal, Osaka, Japan).

\section{Fish and growth trial}

Zero-year-old yellowtail caught in the Tosa Bay, Kochi, Japan, were reared at an aquaculture station (Konan, Kochi, Japan) in indoor tanks supplied with a continuous flow of filtered seawater and fed a commercial diet (Skretting, Fukuoka, Japan). Fish (mean initial weight $=19.1 \mathrm{~g}$ ) were distributed randomly into 18 round fiber-reinforced plastic tanks (1100-liter capacity) with 25 fish per tank (in triplicate). The experiment was conducted from 12 June 2015 to 7 August 2015. The water temperature during the trial ranged from 20.4 to $25.0^{\circ} \mathrm{C}$. During the experiment, fish were hand-fed the experimental diets once a day until satiation. The fish were counted and weighed at the start of the experiment and at 4 and 8 weeks after $48 \mathrm{~h}$ of fasting. At the start ( 5 fish) and at 8 weeks ( 10 fish per tank), fish were sampled after euthanizing with an anesthetic overdose of 2-phenoxyethanol ( $2 \mathrm{~mL} / \mathrm{L}$ ) (Nacalai Tesque Inc., Kyoto, Japan) dissolved in seawater. Five fish each from the initial and final sampling were used for whole-body composition measurements. Blood was collected using a syringe from the caudal vein and the liver was removed from the same five fish. The pooled whole fish and liver samples were used for the determination of the proximate composition and fatty acid composition. Blood samples were left to stand at $4{ }^{\circ} \mathrm{C}$ for $6 \mathrm{~h}$, after which sera were separated by centrifugation at $10,000 \times g$ for $10 \mathrm{~min}$ and stored at $-30{ }^{\circ} \mathrm{C}$ until use in subsequent analyses. Whole fish and liver samples were stored at $-30{ }^{\circ} \mathrm{C}$ until subsequent analysis.

\section{Growth parameters}

Weight gain, specific growth rate (SGR), feed efficiency, protein efficiency ratio, daily feed intake, condition factor, and survival rate were calculated using the formulas shown in the footnote of Table 3.

\section{Chemical composition analysis}

The proximate composition of the diet, whole fish, and liver were analyzed. Crude protein was determined using the semi-micro-Kjeldahl method $(\mathrm{N} \times 6.25)$. Crude lipid was determined by the methanol-chloroform extraction method (Folch et al. 1957). Moisture was determined by drying the 
Table 1 Ingredients and composition of experimental diets for juvenile yellowtail Seriola quinqueradiata

\begin{tabular}{|c|c|c|c|c|c|c|}
\hline \multirow[t]{2}{*}{ Ingredients } & \multicolumn{6}{|c|}{ Diets } \\
\hline & FO & AM0 & AM1 & AM2 & AM3 & AM4 \\
\hline Fish meal & 690 & 690 & 690 & 690 & 690 & 690 \\
\hline Krill meal & 30 & 30 & 30 & 30 & 30 & 30 \\
\hline Algal meal ${ }^{\mathrm{a}}$ & 0 & 0 & 10 & 20 & 30 & 40 \\
\hline Pollack liver oil ${ }^{\mathrm{b}}$ & 50 & 0 & 0 & 0 & 0 & 0 \\
\hline Canola oil $^{\mathrm{c}}$ & 0 & 20 & 13 & 12 & 9 & 5 \\
\hline Palm oil $^{\mathrm{d}}$ & 0 & 30 & 32 & 26 & 21 & 17 \\
\hline Wheat flour & 70 & 70 & 70 & 60 & 50 & 40 \\
\hline$\alpha$-starch & 70 & 70 & 70 & 77 & 85 & 93 \\
\hline Cellulose & 5 & 5 & 0 & 0 & 0 & 0 \\
\hline Vitamin mixture ${ }^{\mathrm{e}}$ & 25 & 25 & 25 & 25 & 25 & 25 \\
\hline Mineral mixture ${ }^{f}$ & 25 & 25 & 25 & 25 & 25 & 25 \\
\hline $\mathrm{CMC}^{7}$ & 25 & 25 & 25 & 25 & 25 & 25 \\
\hline Guar gum & 10 & 10 & 10 & 10 & 10 & 10 \\
\hline \multicolumn{7}{|c|}{ Proximate composition (g/kg) (dry matter basis) } \\
\hline Crude protein & 491 & 484 & 490 & 485 & 488 & 486 \\
\hline Crude lipid & 134 & 136 & 139 & 136 & 130 & 134 \\
\hline Ash & 138 & 135 & 133 & 134 & 133 & 129 \\
\hline
\end{tabular}

${ }^{a}$ All-G Rich (Alltech Inc. Lexington, KY) (crude lipid 71.7\%)

${ }^{\mathrm{b}}$ Riken feed oil $\Omega$ (Eiken Shoji Co. Ltd., Tokyo, Japan)

${ }^{\mathrm{c}}$ Canola oil (Ueda Seiyu Co. Ltd., Hyogo, Japan)

${ }^{\mathrm{d}}$ Palm olein $100 \mathrm{~N}$ (Fuji oil Co. Ltd., Osaka, Japan)

${ }^{\mathrm{e}}$ Vitamins (mg/100 g dry diet): thiamine $\mathrm{HNO}_{3}, 2.2$; pyridoxine $\mathrm{HCl}, 2.3$; nicotinic acid, 9.6; inositol, 60; folic acid, 2.4; choline chloride, 300; ascorbic acid 2-phosphate magnesium ester, 20; riboflavin, 2.2; calcium pantothenate, 7.2; biotin, 0.14; cyanocobalamin, 0.04; vitamin A oil, $1100 \mathrm{IU}$; $\alpha$-tocopherol, 20; $\alpha$-cellulose, rest (modified from Takeda et al. 1989)

${ }^{\mathrm{f}}$ Minerals (mg/100 g dry diet): $\mathrm{KH}_{2} \mathrm{PO}_{4}, 412 ; \mathrm{Ca}\left(\mathrm{H}_{2} \mathrm{PO}_{4}\right)_{2}$, 618; calcium lactate, 282; $\mathrm{C}_{4} \mathrm{H}_{2} \mathrm{FeO}_{4} 160$; $\mathrm{ZnSO}_{4}, 17.65 ; \mathrm{MnSO}_{4}, 9.1 ; \mathrm{CuSO}_{4}, 1.55 ; \mathrm{CoCl}_{2}, 0.05 ; \mathrm{Ca}\left(\mathrm{IO}_{3}\right)_{2}, 0.15 ; \alpha$-cellulose, rest (Masumoto et al. 1996)

${ }^{7}$ Carboxymethyl cellulose sodium salt sample for $10 \mathrm{~h}$ at $110^{\circ} \mathrm{C}$. Ash was determined by combustion for $5 \mathrm{~h}$ at $550{ }^{\circ} \mathrm{C}$.

\section{Blood chemical analysis}

Total cholesterol, triglyceride, glucose, and total protein concentrations in the serum were determined using an automatic analyzer (Fuji Dri-chem 3500 V, Fuji Photo Film, Tokyo, Japan).

\section{Fatty acid analysis}

The lipid source and lipids present in the experimental diets, whole fish, and liver were extracted using a chloroform-methanol $(2: 1 \mathrm{v} / \mathrm{v})$ mixture, according to the method described by Folch et al. (1957), with modifications. The lipid was desiccated under vacuum following solvent removal by evaporation and stored at $-30{ }^{\circ} \mathrm{C}$ until subsequent treatment. The lipid was saponified using $2 \mathrm{~N}$ $\mathrm{NaOH}$ in methanol and esterified using $2 \mathrm{~N}$ methanolic $\mathrm{HCl}$. The fatty acid methyl esters thus obtained were dissolved in $n$-hexane and analyzed using a GC-4000 gas chromatograph (GL Sciences Inc., Tokyo, Japan) equipped with an open-tubular capillary column (InertCap Pure-WAX, $30 \mathrm{~m} \times 0.25 \mathrm{~mm}$ internal diameter, $0.25 \mu \mathrm{m}$ film thickness, GL Sciences Inc.). The oven temperature was initially set at $160{ }^{\circ} \mathrm{C}$ and programmed to reach a final temperature of $250{ }^{\circ} \mathrm{C}$ at a rate of $3{ }^{\circ} \mathrm{C} / \mathrm{min}$. Finally, the oven temperature was maintained at $250{ }^{\circ} \mathrm{C}$ for $5 \mathrm{~min}$. The injection and detection temperatures were $250{ }^{\circ} \mathrm{C}$. A flame ionization detector was used to detect fatty acid methyl esters. Nitrogen was used as the carrier gas.

\section{Nutrient and fatty acid retention}

The retention rate of each nutrient and fatty acid was calculated using the following formulas: 
Nutrient retention $(\%)=[$ gained nutrient $(\mathrm{g}) /$ nutrient intake $(\mathrm{g})] \times 100$

Fatty acid retention $(\%)=[$ gained fatty acid content $(\mathrm{g}) /$ fatty acid intake $(\mathrm{g})] \times 100$

\section{Statistical analysis}

All data were analyzed using one-way analysis of variance, followed by Tukey's multiple comparison test, using GraphPad Prism 7 (GraphPad Software Inc., San Diego, CA, USA). Differences between groups were significant at $P<0.05$. Second-order polynomial regression analysis of the relationship between SGR and dietary fatty acid (DHA/n-3 LC-PUFA) levels was used to estimate the optimal dietary fatty acid level in the diets of juvenile yellowtail.

\section{Results}

\section{Fatty acid composition of the experimental diets}

The fatty acid composition of the experimental diets are shown in Table 2. The pollack liver oil used in the FO diet contained essential fatty acids, including various classes of polyunsaturated fatty acids. Canola oil mainly contained oleic acid (18:1n-9) (67.7\%), followed by palmitic acid (16:0), linoleic acid (18:2n-6), and linolenic acid (18:3n-3). Palm oil mainly comprised palmitic acid (40.6\%) and oleic acid $(44.0 \%)$. The main fatty acids of AM were palmitic acid (50.0\%) and DHA (22:6n-3) (23.6\%) in lipid (crude lipid level $=71.7 \%$ ).

The myristic acid (14:0) level in the non-FO diets was lower than that in the FO diet. Palmitic acid and linoleic acid levels were higher in the non-FO diets than in the FO diet. The palmitic acid level increased with increasing dietary AM concentration. The 20:1n-(9/11) and EPA (20:5n-3) levels were lower in the non-FO diets. DHA level was the lowest in the AM0 diet and increased with AM concentration; AM2 and the FO diet had the same level of DHA. The ratio of $n-3$ and $n-6$ was low in the non-FO diets but increased with dietary level.
Table 2 Fatty acid composition of lipid source and experimental diets

\begin{tabular}{|c|c|c|c|c|c|c|c|c|c|c|}
\hline \multirow[t]{2}{*}{ Fatty acid ( $\%$ fatty acid) } & \multicolumn{4}{|c|}{ Lipid source } & \multicolumn{6}{|l|}{ Diets } \\
\hline & PLO & $\mathrm{CO}$ & $\mathrm{PO}$ & $\mathrm{AM}$ & FO & AM0 & AM1 & AM2 & AM3 & AM4 \\
\hline 14:0 (Myristic acid) & 6.2 & ND & 0.8 & ND & 6.0 & 3.4 & 4.7 & 4.4 & 4.1 & 4.6 \\
\hline 16:0 (Palmitic acid) & 13.4 & 3.1 & 40.6 & 50.0 & 16.8 & 23.1 & 29.1 & 30.7 & 30.6 & 34.3 \\
\hline $16: 1 n-7$ & 6.1 & ND & ND & ND & 6.4 & 3.7 & 4.1 & 3.9 & 3.6 & 3.8 \\
\hline 18:0 (Stearic acid) & 1.9 & ND & 4.4 & 1.3 & 2.9 & 3.3 & 3.5 & 3.6 & 3.3 & 3.5 \\
\hline 18:1n-9 (Oleic acid) & 10.0 & 67.7 & 44.0 & ND & 10.2 & 23.0 & 17.4 & 17.6 & 19.2 & 11.6 \\
\hline $18: 1 n-7$ & 3.3 & ND & ND & ND & 3.2 & 9.1 & 1.9 & 2.2 & 2.3 & 1.3 \\
\hline 18:2n-6 (Linoleic acid) & 1.3 & 20.6 & ND & ND & 2.5 & 8.4 & 9.2 & 6.7 & 5.4 & 4.4 \\
\hline $18: 3 n-3$ & 1.0 & 5.8 & 11.2 & ND & 2.5 & 1.9 & 1.8 & 1.3 & 1.0 & 0.7 \\
\hline $18: 4 n-3$ & 3.9 & ND & ND & ND & 0.2 & 0.9 & 1.1 & 1.0 & 0.9 & 0.0 \\
\hline $20: 1 \mathrm{n}-(9 / 11)$ & 13.3 & 1.2 & ND & ND & 7.6 & 0.7 & 0.7 & 0.5 & 0.4 & 0.5 \\
\hline $20: 4 n-6$ & 0.5 & ND & ND & ND & 0.7 & 0.6 & 0.6 & 0.7 & 0.7 & 0.9 \\
\hline $20: 5 n-3$ (EPA) & 8.9 & ND & ND & 0.3 & 11.8 & 7.4 & 7.7 & 7.8 & 7.6 & 8.2 \\
\hline $22: 5 n-3$ & 1.9 & ND & ND & ND & 1.6 & 1.0 & 0.9 & 1.1 & 1.1 & 1.9 \\
\hline 22:6n-3 (DHA) & 8.8 & ND & ND & 23.6 & 12.4 & 7.6 & 9.1 & 12.4 & 13.8 & 16.9 \\
\hline SFA & 21.5 & 3.1 & 45.8 & 51.3 & 25.6 & 29.8 & 37.3 & 38.6 & 38.0 & 42.3 \\
\hline MUFA & 32.7 & 68.9 & 44.0 & 0 & 27.5 & 36.5 & 24.1 & 24.2 & 25.5 & 17.2 \\
\hline n-3 LC-PUFA & 19.6 & 0 & 0 & 23.9 & 25.8 & 16.0 & 17.8 & 21.2 & 22.5 & 27.0 \\
\hline$n-3 / n-6$ & 13.6 & NA & NA & NA & 8.7 & 2.1 & 2.1 & 3.2 & 4.0 & 5.3 \\
\hline \multicolumn{11}{|l|}{ Fatty acid (\% diet) } \\
\hline 22:6n-3 (DHA) & & & & & 1.66 & 1.04 & 1.27 & 1.69 & 1.79 & 2.27 \\
\hline n-3 LC-PUFA & & & & & 3.46 & 2.17 & 2.48 & 2.89 & 2.92 & 3.62 \\
\hline
\end{tabular}

$N D$ not detected, $N A$ non-available, $P L O$ pollack liver oil, $C O$ canola oil, $P O$ palm oil, SFA saturated fatty acid, MUFA monounsaturated fatty acid, $n-3 L C$-PUFA long-chain polyunsaturated fatty acid 
Table 3 Growth performance and feed utilization of juvenile yellowtail Seriola quinqueradiata fed the experimental diets for 8 weeks

\begin{tabular}{|c|c|c|c|c|c|c|}
\hline & \multicolumn{6}{|l|}{ Dietary group } \\
\hline & FO & AM0 & AM1 & AM2 & AM3 & AM4 \\
\hline Initial body weight (g) & $19.0 \pm 0.1$ & $19.0 \pm 0.1$ & $19.2 \pm 0.1$ & $19.1 \pm 0.1$ & $19.1 \pm 0.1$ & $19.1 \pm 0.1$ \\
\hline Final body weight (g) & $93.0 \pm 1.3^{\mathrm{ab}}$ & $86.5 \pm 2.0^{\mathrm{a}}$ & $91.8 \pm 3.1^{\mathrm{ab}}$ & $97.8 \pm 2.3^{\mathrm{b}}$ & $96.8 \pm 2.3^{\mathrm{ab}}$ & $94.4 \pm 2.4^{\mathrm{ab}}$ \\
\hline Weight gain $(\%)^{\mathrm{a}}$ & $382.3 \pm 10.7^{\mathrm{ab}}$ & $337.3 \pm 10.4^{\mathrm{a}}$ & $374.4 \pm 19.1^{\mathrm{ab}}$ & $409.4 \pm 15.4^{\mathrm{b}}$ & $405.1 \pm 15.5^{\mathrm{ab}}$ & $389.1 \pm 12.8^{\mathrm{ab}}$ \\
\hline Specific growth rate $(\% / \text { day })^{\mathrm{b}}$ & $2.84 \pm 0.03$ & $2.71 \pm 0.04$ & $2.80 \pm 0.07$ & $2.92 \pm 0.04$ & $2.90 \pm 0.05$ & $2.85 \pm 0.04$ \\
\hline Feed efficiency $(\%)^{\mathrm{c}}$ & $85.7 \pm 1.8^{\mathrm{ab}}$ & $80.4 \pm 1.3^{\mathrm{a}}$ & $85.9 \pm 2.3^{\mathrm{ab}}$ & $91.5 \pm 1.9^{\mathrm{b}}$ & $85.9 \pm 1.7^{\mathrm{ab}}$ & $83.9 \pm 3.4^{\mathrm{ab}}$ \\
\hline Protein efficiency ratio $^{\mathrm{d}}$ & $1.75 \pm 0.04$ & $1.66 \pm 0.03$ & $1.75 \pm 0.05$ & $1.89 \pm 0.04$ & $1.76 \pm 0.03$ & $1.73 \pm 0.07$ \\
\hline Daily feed intake (\% BW/day) ${ }^{\mathrm{e}}$ & $3.47 \pm 0.08$ & $3.52 \pm 0.03$ & $3.44 \pm 0.07$ & $3.29 \pm 0.07$ & $3.47 \pm 0.04$ & $3.52 \pm 0.11$ \\
\hline Condition factor ${ }^{\mathrm{f}}$ & $1.43 \pm 0.00$ & $1.46 \pm 0.02$ & $1.47 \pm 0.01$ & $1.47 \pm 0.01$ & $1.46 \pm 0.02$ & $1.48 \pm 0.03$ \\
\hline Survival rate $(\%)^{\mathrm{g}}$ & $93.3 \pm 3.5$ & $90.7 \pm 4.8$ & $94.7 \pm 3.5$ & $97.3 \pm 1.3$ & $98.7 \pm 1.3$ & $97.3 \pm 1.3$ \\
\hline
\end{tabular}

Values are the mean \pm SEM of triplicate tanks. Different superscripts indicate significant difference by one-way ANOVA, followed by Tukey's multiple range test $(P<0.05)$

${ }^{a}$ Weight gain $=($ final total body weight - initial total body weight $) /$ initial body weight $\times 100$

${ }^{\mathrm{b}}$ Specific growth rate $=100 \times(\ln$ final mean weight $-\ln$ initial mean weight $) /$ trial days

${ }^{\mathrm{c}}$ Feed efficiency $=($ final total body weight - initial total body weight $) /$ total dry feed $\times 100$

${ }^{\mathrm{d}}$ Protein efficiency ratio $=$ weight gain/total protein intake

${ }^{\mathrm{e}}$ Daily feeding intake $=100 \times \mathrm{g}$ dry feed intake/[(initial fish number + final fish number $) / 2 \times(\mathrm{g}$ initial average body weight $+\mathrm{g}$ final average body weight)/2]/feeding days

${ }^{\mathrm{f}}$ Condition factor $=$ individual body weight/body length ${ }^{3} \times 100$

${ }^{\mathrm{g}}$ Survival rate $=$ final fish number/initial fish number $\times 100$

\section{Growth performance}

The growth performance at completion of the feeding trial is shown in Table 3. Final body weight, weight gain, and feed efficiency were significantly lower in the AM0 group and significantly higher in the AM2 group. Other dietary groups did not show significant differences in body weight and weight gain. For the SGR and protein efficiency ratio, no significant differences were observed in any of the dietary groups; however, similar trends were observed in the final body weight, weight gain, and feed efficiency. No significant differences in any of the dietary groups were observed in the daily feed intake, condition factor, and survival rate.

\section{Proximate composition of whole body and liver}

The proximate composition of the whole body and liver are shown in Table 4. No significant differences among the dietary groups were observed with regard to whole-body composition. The crude protein level in liver was the lowest
Table 4 Whole-body and liver composition (wet weight basis) of juvenile yellowtail Seriola quinqueradiata fed the experimental diets for 8 weeks

\begin{tabular}{|c|c|c|c|c|c|c|c|}
\hline & \multicolumn{7}{|c|}{ Dietary group } \\
\hline & Initial & FO & AM0 & AM1 & AM2 & AM3 & AM4 \\
\hline \multicolumn{8}{|c|}{ Whole-body composition (\%) } \\
\hline Moisture & 77.2 & $75.9 \pm 0.4$ & $75.9 \pm 0.3$ & $75.7 \pm 0.2$ & $75.8 \pm 0.5$ & $75.7 \pm 0.4$ & $75.9 \pm 1.1$ \\
\hline Crude protein & 21.2 & $17.9 \pm 0.1$ & $18.5 \pm 0.7$ & $18.0 \pm 0.2$ & $17.3 \pm 0.1$ & $17.9 \pm 0.7$ & $17.4 \pm 0.6$ \\
\hline Crude lipid & 2.5 & $3.6 \pm 0.2$ & $3.3 \pm 0.1$ & $3.3 \pm 0.1$ & $3.7 \pm 0.1$ & $3.6 \pm 0.1$ & $3.5 \pm 0.2$ \\
\hline Ash & 3.7 & $3.3 \pm 0.1$ & $3.2 \pm 0.1$ & $3.3 \pm 0.1$ & $3.0 \pm 0.2$ & $3.3 \pm 0.2$ & $3.4 \pm 0.1$ \\
\hline \multicolumn{8}{|c|}{ Liver composition (\%) } \\
\hline Moisture & & $73.2 \pm 0.4$ & $72.1 \pm 2.0$ & $73.5 \pm 0.3$ & $72.9 \pm 0.3$ & $73.8 \pm 0.6$ & $73.1 \pm 0.1$ \\
\hline Crude protein & & $18.1 \pm 0.8^{\mathrm{ab}}$ & $18.9 \pm 0.2^{\mathrm{ab}}$ & $18.9 \pm 0.1^{\mathrm{ab}}$ & $17.4 \pm 0.1^{\mathrm{a}}$ & $18.7 \pm 0.5^{\mathrm{ab}}$ & $20.0 \pm 1.4^{\mathrm{b}}$ \\
\hline Crude lipid & & $3.9 \pm 0.4^{\mathrm{ab}}$ & $4.4 \pm 0.1^{\mathrm{b}}$ & $3.1 \pm 0.3^{\mathrm{a}}$ & $3.0 \pm 0.1^{\mathrm{a}}$ & $4.2 \pm 0.2^{\mathrm{ab}}$ & $3.6 \pm 0.3^{\mathrm{ab}}$ \\
\hline Ash & & $2.8 \pm 0.5$ & $2.2 \pm 0.2$ & $1.9 \pm 0.3$ & $2.2 \pm 0.2$ & $2.1 \pm 0.1$ & $2.2 \pm 0.4$ \\
\hline
\end{tabular}

Values are the mean \pm SEM of triplicate tanks except initial. Different superscripts indicate significant difference by one-way ANOVA, followed by Tukey's multiple range test $(P<0.05)$. Statistical analysis was performed without the initial group 
in the AM2 group and significantly different from that in the AM4 group. With regard to the crude lipid level of the liver, the AM1 and AM2 groups showed the lowest values, which were significantly different from that in the AM0 group. There was no significant difference in moisture and ash content of the liver.

\section{Estimation of requirements}

Second-order polynomial regression analysis was used to estimate the optimal dietary fatty acid (DHA/n-3 LC-PUFA) level for juvenile yellowtail (Fig. 1). Second-order polynomial regression curves $\left[Y=-0.265 X^{2}+0.994 X+1.961\left(R^{2}=0.486\right)\right.$ and $\left.Y=-0.250 X^{2}+1.540 X+0.533\left(R^{2}=0.502\right)\right]$ were used to determine the optimal point for the maximum SGR. The
Fig. 1 a Relationship between dietary docosahexaenoic acid (DHA) level and specific growth rate of juvenile yellowtail Seriola quinqueradiata as fit by second-order polynomial regression analysis. b Relationship between dietary n-3 LCPUFA level and specific growth rate of juvenile yellowtail $S$. quinqueradiata as fit by secondorder polynomial regression analysis

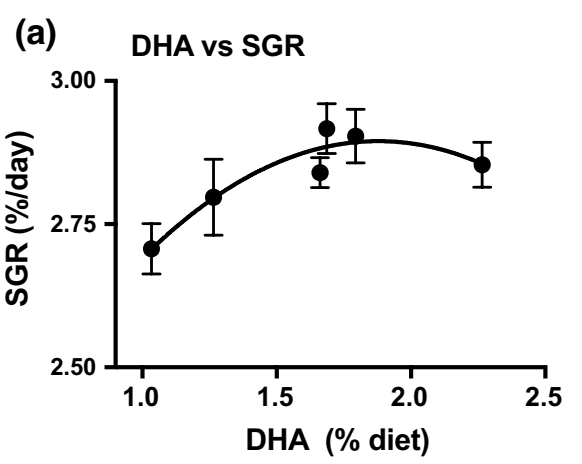

$$
\begin{aligned}
& y=-0.265 x^{2}+0.994 x+1.961 \\
& R^{2}=0.486 \\
& \text { Max: } 1.88
\end{aligned}
$$

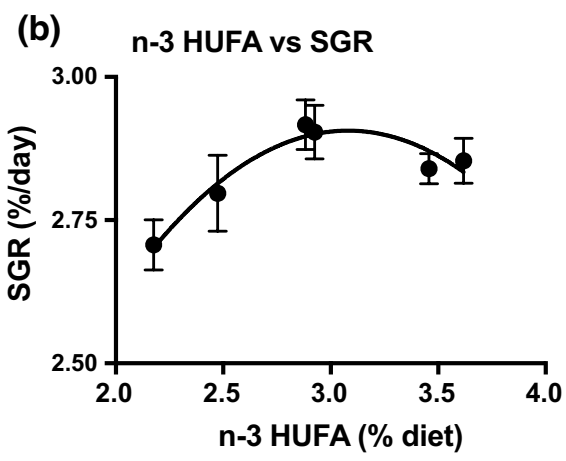

\begin{tabular}{|c|c|c|c|c|c|c|c|}
\hline \multirow[t]{2}{*}{ Fatty acid (area \%) } & \multicolumn{7}{|c|}{ Dietary group } \\
\hline & Initial & FO & AM0 & AM1 & AM2 & AM3 & AM4 \\
\hline 14:0 (Myristic acid) & 2.7 & $4.9 \pm 0.0^{c}$ & $3.4 \pm 0.0^{\mathrm{ab}}$ & $3.3 \pm 0.1^{\mathrm{a}}$ & $3.8 \pm 0.1^{b}$ & $3.5 \pm 0.1^{\mathrm{ab}}$ & $3.4 \pm 0.1^{\mathrm{ab}}$ \\
\hline 16:0 (Palmitic acid) & 18.1 & $20.0 \pm 0.4^{\mathrm{a}}$ & $22.4 \pm 0.7^{\mathrm{ab}}$ & $21.9 \pm 1.2^{\mathrm{a}}$ & $25.5 \pm 0.5^{\mathrm{b}}$ & $22.9 \pm 0.7^{\mathrm{ab}}$ & $24.8 \pm 0.3^{\mathrm{b}}$ \\
\hline $16: 1 n-7$ & 2.6 & $5.3 \pm 0.1^{\mathrm{a}}$ & $3.8 \pm 0.0^{\mathrm{bc}}$ & $3.6 \pm 0.2^{\mathrm{c}}$ & $4.1 \pm 0.1^{\mathrm{b}}$ & $3.8 \pm 0.1^{\mathrm{bc}}$ & $3.6 \pm 0.0^{\mathrm{bc}}$ \\
\hline 18:0 (Stearic acid) & 8.2 & $5.0 \pm 0.2$ & $5.5 \pm 0.1$ & $5.2 \pm 0.3$ & $6.1 \pm 0.2$ & $5.5 \pm 0.1$ & $6.6 \pm 0.8$ \\
\hline 18:1n-9 (Oleic acid) & 14.9 & $16.0 \pm 0.9^{\mathrm{a}}$ & $28.4 \pm 0.6^{\mathrm{b}}$ & $24.9 \pm 1.2^{\mathrm{b}}$ & $17.2 \pm 0.5^{\mathrm{a}}$ & $19.6 \pm 1.1^{\mathrm{a}}$ & $17.9 \pm 0.3^{\mathrm{a}}$ \\
\hline $18: 1 n-7$ & 3.3 & $3.7 \pm 0.5^{\mathrm{ab}}$ & $3.2 \pm 0.3^{\mathrm{a}}$ & $3.9 \pm 0.8^{\mathrm{ab}}$ & $3.9 \pm 0.6^{\mathrm{ab}}$ & $5.8 \pm 0.1^{\mathrm{b}}$ & $4.7 \pm 0.3^{\mathrm{ab}}$ \\
\hline $18: 2 n-6$ (Linoleic acid) & 2.6 & $4.0 \pm 0.4^{\mathrm{a}}$ & $7.8 \pm 0.2^{\mathrm{bc}}$ & $7.3 \pm 0.2^{\mathrm{bc}}$ & $8.0 \pm 0.1^{\mathrm{c}}$ & $6.2 \pm 0.3^{b}$ & $5.0 \pm 0.1^{\mathrm{a}}$ \\
\hline $18: 3 n-3$ & 0.3 & $0.7 \pm 0.1^{\mathrm{a}}$ & $1.3 \pm 0.0^{\mathrm{c}}$ & $1.2 \pm 0.0^{\mathrm{c}}$ & $1.3 \pm 0.0^{c}$ & $1.0 \pm 0.0^{\mathrm{b}}$ & $0.6 \pm 0.0^{\mathrm{a}}$ \\
\hline $18: 4 n-3$ & 0.2 & $1.3 \pm 0.0$ & $1.1 \pm 0.4$ & $0.7 \pm 0.0$ & $0.8 \pm 0.0$ & $0.7 \pm 0.1$ & $1.3 \pm 0.0$ \\
\hline $20: 1 n-(9 / 11)$ & 2.0 & $5.8 \pm 0.3^{\mathrm{b}}$ & $2.7 \pm 1.3^{\mathrm{a}}$ & $1.2 \pm 0.1^{\mathrm{a}}$ & $0.9 \pm 0.1^{\mathrm{a}}$ & $1.1 \pm 0.5^{\mathrm{a}}$ & $0.6 \pm 0.0^{\mathrm{a}}$ \\
\hline $20: 4 n-6$ & 1.5 & $0.7 \pm 0.1^{\mathrm{ab}}$ & $0.7 \pm 0.2^{\mathrm{ab}}$ & $0.5 \pm 0.2^{\mathrm{ab}}$ & $1.0 \pm 0.11^{\mathrm{b}}$ & $1.0 \pm 0.0^{\mathrm{b}}$ & $0.4 \pm 0.0^{\mathrm{a}}$ \\
\hline 20:5n-3 (EPA) & 4.6 & $6.1 \pm 0.8$ & $5.1 \pm 0.1$ & $4.9 \pm 0.3$ & $5.3 \pm 0.1$ & $5.2 \pm 0.2$ & $5.3 \pm 0.1$ \\
\hline $22: 5 n-3$ & 2.0 & $2.1 \pm 0.1^{b}$ & $1.7 \pm 0.1^{\mathrm{ab}}$ & $1.5 \pm 0.2^{\mathrm{a}}$ & $1.6 \pm 0.0^{\mathrm{a}}$ & $1.6 \pm 0.1^{\mathrm{a}}$ & $2.6 \pm 0.0^{c}$ \\
\hline 22:6n-3 (DHA) & 26.2 & $14.3 \pm 1.0^{\mathrm{ab}}$ & $11.4 \pm 0.3^{\mathrm{a}}$ & $12.1 \pm 0.9^{\mathrm{a}}$ & $13.5 \pm 0.8^{\mathrm{ab}}$ & $16.6 \pm 0.5^{\mathrm{b}}$ & $20.6 \pm 0.3^{\mathrm{c}}$ \\
\hline SFA & 29.0 & $29.8 \pm 0.3^{\mathrm{a}}$ & $31.3 \pm 0.5^{\mathrm{a}}$ & $30.5 \pm 1.6^{\mathrm{a}}$ & $35.4 \pm 0.7^{b}$ & $31.8 \pm 0.6^{\mathrm{a}}$ & $34.1 \pm 0.3^{b}$ \\
\hline MUFA & 22.8 & $30.7 \pm 1.0^{\mathrm{bc}}$ & $38.1 \pm 1.0^{\mathrm{d}}$ & $33.6 \pm 0.4^{\mathrm{c}}$ & $26.1 \pm 0.3^{\mathrm{a}}$ & $30.2 \pm 0.5^{\mathrm{b}}$ & $26.7 \pm 0.2^{\mathrm{a}}$ \\
\hline n-3 LC-PUFA & 32.8 & $22.5 \pm 1.5^{\mathrm{a}}$ & $18.2 \pm 0.5^{\mathrm{a}}$ & $18.5 \pm 1.2^{\mathrm{a}}$ & $20.4 \pm 0.9^{\mathrm{a}}$ & $23.4 \pm 0.3^{\mathrm{b}}$ & $28.5 \pm 0.3^{\mathrm{c}}$ \\
\hline$n-3 / n-6$ & 7.9 & $5.4 \pm 0.8 b$ & $2.4 \pm 0.1^{\mathrm{a}}$ & $2.6 \pm 0.2^{\mathrm{a}}$ & $2.5 \pm 0.1^{\mathrm{a}}$ & $3.4 \pm 0.1^{\mathrm{a}}$ & $5.7 \pm 0.1^{\mathrm{b}}$ \\
\hline
\end{tabular}

$$
\begin{aligned}
& \mathrm{y}=-0.250 \mathrm{x}^{2}+1.540 \mathrm{x}+0.533 \\
& \mathrm{R}^{2}=0.502 \\
& \text { Max: } 3.08
\end{aligned}
$$

Table 5 Whole-body fatty acid composition in juvenile yellowtail Seriola quinqueradiata fed the experimental diet for 8 weeks

Values are the mean \pm SEM of triplicate tanks. Values in the same line with different superscripts are significantly different $(P<0.05)$. Statistical analysis was performed without the initial group. SFA saturated fatty acid, MUFA monounsaturated fatty acid, LC-PUFA long-chain polyunsaturated fatty acid 
results showed that the maxima were at $1.88 \%$ (in diet) for DHA and $3.08 \%$ (in diet) for n-3 LC-PUFA.

\section{Fatty acid composition of whole body and liver}

The fatty acid composition of the whole body is shown in Table 5. Saturated fatty acid composition in the AM2 $(35.4 \pm 0.7 \%)$ and AM4 $(34.1 \pm 0.3 \%)$ groups was significantly higher than that of the other groups (29.8-31.8\%). Palmitic acid, a major saturated fatty acid, was over $20 \%$, with AM2 $(25.5 \pm 0.5 \%)$ and AM4 $(24.8 \pm 0.3 \%)$ groups showing significantly higher values than that of the FO $(20.0 \pm 0.4 \%)$ and AM1 $(21.9 \pm 1.2 \%)$ groups. The highest value of monounsaturated fatty acid (MUFA) was observed in the AM0 group $(38.1 \pm 1.0 \%)$, whereas AM2 $(26.1 \pm 0.3 \%)$ and AM4 $(26.7 \pm 0.2 \%)$ groups had significantly lower values than the other dietary groups. The major MUFA was oleic acid, which was significantly higher in the AM0 $(28.4 \pm 0.6 \%)$ and AM1 $(24.9 \pm 1.2 \%)$ groups than in the other dietary groups (16.0-19.6\%). High levels of linoleic acid were observed in the AM0, AM1, AM2, and AM3 groups $(6.2-8.0 \%)$, significantly higher than the levels in the FO $(4.0 \pm 0.4 \%)$ and AM4 $(5.0 \pm 0.1 \%)$ groups. The $n-3$ LC-PUFA level in the whole body was the highest in the AM4 group (28.5 $\pm 0.3 \%)$ and the lowest in the AM0 group $(18.2 \pm 0.5 \%)$. The levels of EPA, an n-3 LC-PUFA, did not show any significant differences among the dietary groups. The DHA level increased from $11.4 \%$ to $20.6 \%$ with supplementation of AM.

The fatty acid composition of the liver is shown in Table 6. Saturated fatty acid composition did not show any significant differences among the dietary groups (32.2-37.9\%). The level of palmitic acid was over $20 \%$, with the AM2 $(27.2 \pm 0.2 \%)$ and AM4 $(29.5 \pm 1.1 \%)$ groups showing higher values than the other groups (24.1-26.3\%). Linoleic acid in the AM0, AM1, and AM2 groups (3.1-4.8\%) was significantly higher than that in the FO group $(1.3 \pm 0.1 \%)$. For MUFA, no significant differences were found among the dietary groups (10.7-16.5\%). Oleic acid was significantly higher in the AM0 group $(12.6 \pm 1.7 \%)$ than in the FO, AM3, and AM4 groups (6.6-7.9\%). The level of n-3 LC-PUFA showed high abundance in the liver $(>31.7 \%)$ with the highest level observed in the FO group $(42.7 \pm 2.1 \%)$ and the lowest in the AM0 group $(31.7 \pm 3.3 \%)$. The EPA level was significantly lower in the AM3 $(4.8 \pm 0.4 \%)$ and AM4 $(4.7 \pm 0.4 \%)$ groups than in the FO group $(6.9 \pm 0.2 \%)$. The DHA level was the highest in the FO group $(33.6 \pm 2.2 \%)$, which was significantly higher than that in the AM0 group $(23.8 \pm 2.6 \%)$.

The differences in fatty acid concentrations between diet and whole body/liver are shown in Table 7. In the whole body, the concentrations of myristic acid were lower
Table 6 Liver fatty acid composition in juvenile yellowtail Seriola quinqueradiata fed the experimental diet for 8 weeks

\begin{tabular}{|c|c|c|c|c|c|c|}
\hline \multirow[t]{2}{*}{ Fatty acid (area \%) } & \multicolumn{6}{|c|}{ Dietary group } \\
\hline & FO & AM0 & AM1 & AM2 & AM3 & AM4 \\
\hline 14:0 (Myristic acid) & $1.7 \pm 0.1^{\mathrm{b}}$ & $1.0 \pm 0.1^{\mathrm{a}}$ & $0.8 \pm 0.0^{\mathrm{a}}$ & $0.9 \pm 0.1^{\mathrm{a}}$ & $0.9 \pm 0.1^{\mathrm{a}}$ & $0.9 \pm 0.1^{\mathrm{a}}$ \\
\hline 16:0 (Palmitic acid) & $24.1 \pm 0.7$ & $26.0 \pm 1.6$ & $24.7 \pm 1.6$ & $27.2 \pm 0.2$ & $26.3 \pm 2.0$ & $29.5 \pm 1.1$ \\
\hline $16: 1 n-7$ & $1.7 \pm 0.0^{\mathrm{b}}$ & $1.2 \pm 0.1^{\mathrm{a}}$ & $0.9 \pm 0.1^{\mathrm{a}}$ & $1.0 \pm 0.1^{\mathrm{a}}$ & $1.0 \pm 0.1^{\mathrm{a}}$ & $1.1 \pm 0.1^{\mathrm{a}}$ \\
\hline 18:0 (Stearic acid) & $7.6 \pm 0.1$ & $7.7 \pm 0.2$ & $6.7 \pm 0.6$ & $6.7 \pm 0.2$ & $6.2 \pm 0.3$ & $7.5 \pm 0.3$ \\
\hline 18:1n-9 (Oleic acid) & $6.6 \pm 0.2^{\mathrm{a}}$ & $12.6 \pm 1.7^{\mathrm{b}}$ & $8.7 \pm 1.2^{\mathrm{ab}}$ & $8.7 \pm 0.6^{\mathrm{ab}}$ & $7.9 \pm 0.7^{\mathrm{a}}$ & $7.4 \pm 0.8^{\mathrm{a}}$ \\
\hline $18: 1 n-7$ & $2.6 \pm 0.2$ & $2.2 \pm 0.2$ & $1.8 \pm 0.1$ & $1.9 \pm 0.0$ & $1.5 \pm 0.5^{\mathrm{b}}$ & $2.0 \pm 0.1^{\mathrm{ab}}$ \\
\hline 18:2n-6 (Linoleic acid) & $1.3 \pm 0.1^{\mathrm{a}}$ & $4.8 \pm 0.4^{\mathrm{c}}$ & $3.2 \pm 0.5^{\mathrm{b}}$ & $3.1 \pm 0.1^{\mathrm{b}}$ & $2.5 \pm 0.2^{\mathrm{ab}}$ & $2.0 \pm 0.2^{\mathrm{ab}}$ \\
\hline $18: 3 n-3$ & $0.2 \pm 0.0^{\mathrm{a}}$ & $0.5 \pm 0.1^{\mathrm{b}}$ & $0.4 \pm 0.0^{\mathrm{a}}$ & $0.4 \pm 0.0^{\mathrm{a}}$ & $0.3 \pm 0.0^{\mathrm{a}}$ & $0.2 \pm 0.0^{\mathrm{a}}$ \\
\hline $18: 4 n-3$ & $0.3 \pm 0.0^{\mathrm{a}}$ & $0.1 \pm 0.0^{\mathrm{b}}$ & $0.1 \pm 0.0^{\mathrm{b}}$ & $0.1 \pm 0.0^{\mathrm{b}}$ & $0.1 \pm 0.0^{\mathrm{b}}$ & $0.1 \pm 0.0^{\mathrm{b}}$ \\
\hline $20: 1 n-(9 / 11)$ & $2.3 \pm 0.3^{\mathrm{b}}$ & $0.4 \pm 0.0^{\mathrm{a}}$ & $0.2 \pm 0.0^{\mathrm{a}}$ & $0.2 \pm 0.0^{\mathrm{a}}$ & $0.3 \pm 0.2^{\mathrm{a}}$ & $0.4 \pm 0.2^{\mathrm{a}}$ \\
\hline $20: 4 n-6$ & $2.9 \pm 0.1$ & $2.5 \pm 0.2$ & $2.5 \pm 0.1$ & $2.6 \pm 0.1$ & $2.5 \pm 0.1$ & $2.7 \pm 0.3$ \\
\hline $20: 5 n-3$ (EPA) & $6.9 \pm 0.2^{\mathrm{b}}$ & $5.9 \pm 0.5^{\mathrm{ab}}$ & $5.6 \pm 0.5^{\mathrm{ab}}$ & $5.8 \pm 0.1^{\mathrm{ab}}$ & $4.8 \pm 0.4^{\mathrm{a}}$ & $4.7 \pm 0.4^{\mathrm{a}}$ \\
\hline $22: 5 n-3$ & $2.3 \pm 0.1^{\mathrm{b}}$ & $2.0 \pm 0.2^{\mathrm{b}}$ & $1.6 \pm 0.2^{\mathrm{a}}$ & $1.4 \pm 0.1^{\mathrm{a}}$ & $1.1 \pm 0.1^{\mathrm{a}}$ & $1.1 \pm 0.1^{\mathrm{a}}$ \\
\hline 22:6n-3 (DHA) & $33.6 \pm 2.2^{\mathrm{b}}$ & $23.8 \pm 2.6^{\mathrm{a}}$ & $29.4 \pm 0.5^{\mathrm{ab}}$ & $33.0 \pm 1.2^{\mathrm{b}}$ & $30.8 \pm 1.9^{\mathrm{ab}}$ & $32.3 \pm 2.1^{\mathrm{ab}}$ \\
\hline SFA & $33.4 \pm 1.4$ & $34.5 \pm 2.0$ & $32.2 \pm 2.2$ & $34.8 \pm 0.3$ & $33.4 \pm 2.5$ & $37.9 \pm 1.1$ \\
\hline MUFA & $13.2 \pm 0.7$ & $16.5 \pm 2.0$ & $11.6 \pm 1.4$ & $11.7 \pm 0.7$ & $10.7 \pm 1.4$ & $11.0 \pm 1.3$ \\
\hline n-3 LC-PUFA & $42.7 \pm 2.1^{\mathrm{b}}$ & $31.7 \pm 3.3^{\mathrm{a}}$ & $36.5 \pm 1.2^{\mathrm{ab}}$ & $40.2 \pm 1.1^{\mathrm{ab}}$ & $36.6 \pm 2.3^{\mathrm{ab}}$ & $38.1 \pm 2.6^{\mathrm{ab}}$ \\
\hline$n-3 / n-6$ & $10.3 \pm 0.3^{c}$ & $4.5 \pm 0.5^{\mathrm{a}}$ & $6.5 \pm 0.5^{\mathrm{b}}$ & $7.1 \pm 0.3^{\mathrm{b}}$ & $7.5 \pm 0.4^{\mathrm{b}}$ & $8.1 \pm 0.3^{\mathrm{b}}$ \\
\hline
\end{tabular}

Values are the mean \pm SEM of triplicate tanks. Values in the same line with different superscripts are significantly different $(P<0.05)$

SFA saturated fatty acid, MUFA monounsaturated fatty acid, LC-PUFA long-chain polyunsaturated fatty acid 
Table 7 Differences between diet and whole-body/liver fatty acid concentrations in juvenile yellowtail Seriola quinqueradiata fed the experimental diet for 8 weeks

\begin{tabular}{|c|c|c|c|c|c|c|c|c|c|c|c|c|}
\hline \multirow[t]{2}{*}{ Fatty acid (\%) } & \multicolumn{6}{|c|}{ Whole body } & \multicolumn{6}{|l|}{ Liver } \\
\hline & FO & AM0 & AM1 & AM2 & AM3 & AM4 & FO & AM0 & AM1 & AM2 & AM3 & AM4 \\
\hline 14:0 (Myristic acid) & -1.1 & 0.0 & -1.4 & -0.5 & -0.6 & -1.2 & -4.3 & -2.4 & -4.0 & -3.5 & -3.2 & -3.7 \\
\hline 16:0 (Palmitic acid) & 3.3 & -0.7 & -7.2 & -5.3 & -7.7 & -9.5 & 7.3 & 2.9 & -4.4 & -3.5 & -4.3 & -4.8 \\
\hline $16: 1 n-7$ & -1.2 & 0.1 & -0.5 & 0.2 & 0.1 & -0.2 & -4.7 & -2.5 & -3.2 & -2.9 & -2.6 & -2.7 \\
\hline 18:0 (Stearic acid) & 2.1 & 2.1 & 1.7 & 2.5 & 2.2 & 3.2 & 4.7 & 4.4 & 3.2 & 3.1 & 3.0 & 4.1 \\
\hline 18:1n-9 (Oleic acid) & 5.8 & 5.4 & 7.5 & -0.4 & 0.3 & 6.3 & -3.6 & -10.4 & -8.7 & -8.9 & -11.4 & -4.2 \\
\hline $18: 1 n-7$ & 0.5 & -5.9 & 2.0 & 1.7 & 3.5 & 3.4 & -0.6 & -6.9 & -0.2 & -0.4 & -0.8 & 0.8 \\
\hline 18:2n-6 (Linoleic acid) & 1.5 & -0.6 & -1.9 & 1.3 & 0.8 & 0.6 & -1.3 & -3.6 & -6.0 & -3.6 & -2.9 & -2.3 \\
\hline $18: 3 n-3$ & -1.7 & -0.6 & -0.6 & 0.0 & 0.0 & -0.1 & -2.2 & -1.4 & -1.4 & -0.9 & -0.7 & -0.5 \\
\hline $18: 4 n-3$ & 1.2 & 0.2 & -0.4 & -0.2 & -0.2 & 1.3 & 0.1 & -0.9 & -1.0 & -0.8 & -0.9 & 0.1 \\
\hline $20: 1 n-(9 / 11)$ & -1.8 & 2.0 & 0.5 & 0.4 & 0.7 & 0.1 & -5.3 & -0.3 & -0.4 & -0.3 & -0.1 & -0.1 \\
\hline $20: 4 n-6$ & -0.1 & 0.1 & -0.1 & 0.3 & 0.3 & -0.5 & 2.2 & 1.9 & 1.9 & 1.9 & 1.7 & 1.8 \\
\hline $20: 5 n-3$ (EPA) & -5.7 & -2.3 & -2.8 & -2.5 & -2.5 & -2.9 & -4.9 & -1.5 & -2.2 & -2.0 & -2.9 & -3.4 \\
\hline $22: 5 n-3$ & 0.5 & 0.7 & 0.5 & 0.5 & 0.6 & 0.7 & 0.6 & 1.0 & 0.6 & 0.4 & 0.0 & -0.8 \\
\hline 22:6n-3 (DHA) & 1.9 & 3.8 & 3.0 & 1.1 & 2.8 & 3.7 & 21.2 & 16.2 & 20.2 & 20.6 & 17.0 & 15.4 \\
\hline SFA & 4.2 & 1.5 & -6.8 & -3.3 & -6.1 & -8.3 & 7.7 & 4.9 & -5.2 & -3.9 & -4.5 & -4.4 \\
\hline MUFA & 3.3 & 1.6 & 9.5 & 1.9 & 4.7 & 9.5 & -14.3 & -20.0 & -12.6 & -12.5 & -14.8 & -6.2 \\
\hline n-3 LC-PUFA & -3.3 & 2.2 & 0.7 & -0.9 & 0.9 & 1.5 & 16.9 & 15.7 & 18.7 & 18.9 & 14.1 & 11.1 \\
\hline
\end{tabular}

Differences between diet and whole-body/liver fatty acid = fatty acid in whole body/liver $(\mathrm{g} / 100 \mathrm{~g})$ - fatty acid in diet $(\mathrm{g} / 100 \mathrm{~g})$. Negative values indicate lower values in whole body/liver compared with the diet. Positive values indicate accumulation in whole body/liver relative to the diet

SFA saturated fatty acid, MUFA monounsaturated fatty acid, $n-3$ LC-PUFA long-chain polyunsaturated fatty acid

in all groups, except in the AM0 group than in the dietary lipid. EPA was lower in all groups than in the dietary lipid. Palmitic acid had lower values in all replacement groups than in the dietary lipid. The difference varied for oleic acid among all dietary groups. Stearic acid (18:0), DHA, and MUFA were higher in all groups than in the dietary lipid.

In the liver, myristic acid, palmitoleic acid (16:1n-7), vaccenic acid (18:1n-7), oleic acid, linoleic acid, linolenic acid, EPA, and MUFA were lower in all groups than in the dietary lipid. Stearic acid, DHA, and n-3 LC-PUFA were higher in all groups than in the dietary lipid.

\section{Retention of protein, lipids, and fatty acids}

Protein retention $(27.4 \%$ to $30.1 \%)$ and lipid retention did not show any significant differences among all dietary groups (Table 8, Fig. 2a). Palmitic acid showed high retention in the $\mathrm{FO}$ and $\mathrm{AM} 0$ groups and low retention in the AM1 and AM4 groups (Fig. 2b). For oleic acid, high retention was observed in the FO and AM4 groups and the lowest retention was confirmed in the AM2 group (Fig. 2c). Linoleic acid showed high retention in the FO and AM2 groups, which was significantly higher than that in the AM0 and AM1 groups (Fig. 2c). No significant differences were observed in the retention of EPA and DHA among the dietary groups (Fig. 2d, e).

\section{Blood chemical analyses}

Serum concentrations of glucose, total cholesterol, and triglyceride did not show any significant differences among the dietary groups (Table 9). The AM0 group had the highest total protein concentration, which was significantly higher than that of the FO group.
Table 8 Retention of protein and lipid of juvenile yellowtail Seriola quinqueradiata fed the experimental diets for 8 weeks

\begin{tabular}{lllllll}
\hline Retention (\%) & \multicolumn{5}{l}{ Dietary group } & \\
\cline { 2 - 6 } & FO & VO & AM1 & AM2 & AM3 & AM4 \\
\hline Protein & $29.6 \pm 0.8$ & $29.3 \pm 1.4$ & $29.9 \pm 0.3$ & $30.1 \pm 1.6$ & $27.4 \pm 2.3$ & $28.4 \pm 2.5$ \\
Lipid & $24.9 \pm 1.8$ & $21.2 \pm 0.9$ & $21.9 \pm 1.5$ & $27.3 \pm 1.9$ & $25.9 \pm 1.0$ & $23.3 \pm 1.2$ \\
\hline
\end{tabular}

Values are the mean \pm SEM of triplicate tanks. No significant difference was observed $(P<0.05)$ 

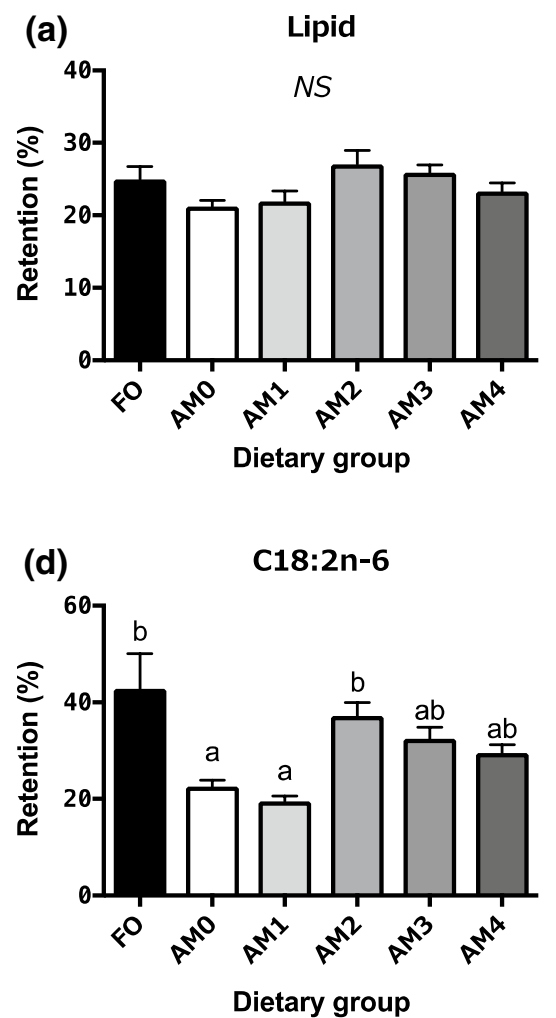
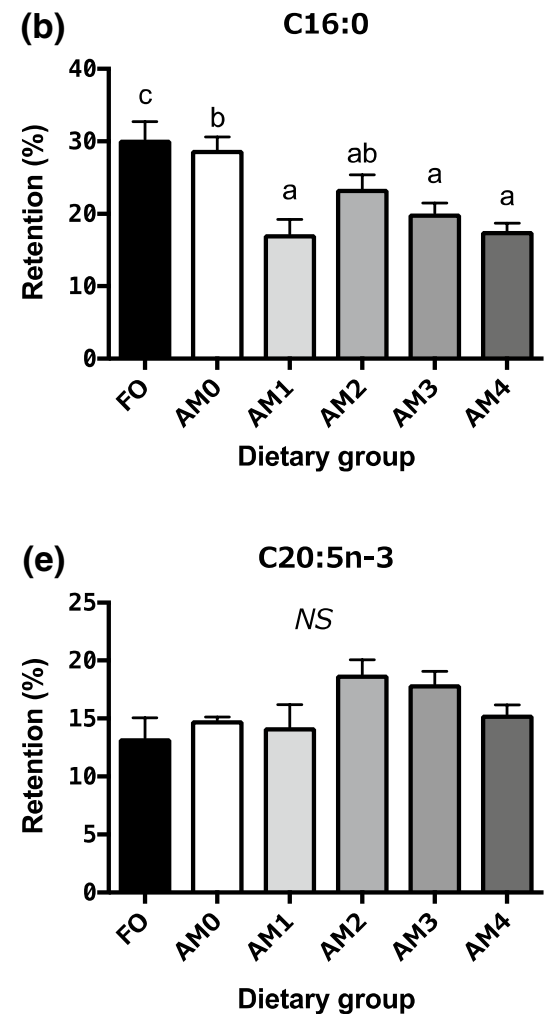
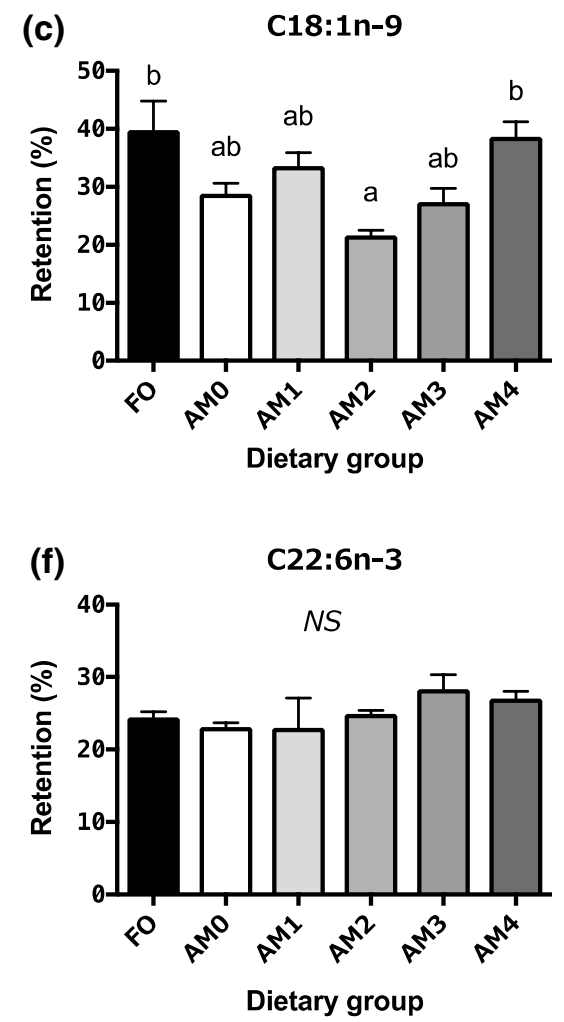

Fig. 2 The retention of a lipid, $\mathbf{b}$ palmitic acid, $\mathbf{c}$ oleic acid, $\mathbf{d}$ linoleic acid, e eicosapentaenoic acid (EPA), and $\mathbf{f}$ docosahexaenoic acid (DHA). Different alphabets in each graph indicate significant differ- ence by one-way analysis of variance, followed by Tukey's multiple range test $(P<0.05)$

Table 9 Concentration of serum components of juvenile yellowtail Seriola quinqueradiata fed the experimental diets for 8 weeks

\begin{tabular}{lcccccc}
\hline Serum components & Dietary group & & & \\
\cline { 2 - 6 } & FO & AM0 & AM1 & AM2 & AM3 & AM4 \\
\hline Total protein $(\mathrm{g} / 100 \mathrm{ml})$ & $3.50 \pm 0.09^{\mathrm{a}}$ & $4.14 \pm 0.03^{\mathrm{b}}$ & $3.77 \pm 0.25^{\text {ab }}$ & $3.39 \pm 0.08^{\mathrm{a}}$ & $3.38 \pm 0.10^{\mathrm{a}}$ & $3.46 \pm 0.12^{\mathrm{a}}$ \\
Glucose $(\mathrm{mg} / 100 \mathrm{ml})$ & $131.7 \pm 7.0$ & $140.7 \pm 14.0$ & $150.3 \pm 12.7$ & $122.1 \pm 12.0$ & $151.7 \pm 3.7$ & $143.0 \pm 3.7$ \\
Total cholesterol $(\mathrm{mg} / 100 \mathrm{ml})$ & $248.2 \pm 24.7$ & $264.2 \pm 2.2$ & $241.2 \pm 8.3$ & $234.6 \pm 28.5$ & $266.7 \pm 2.7$ & $248.1 \pm 6.6$ \\
Triglyceride $(\mathrm{mg} / 100 \mathrm{ml})$ & $92.3 \pm 5.3$ & $101.1 \pm 9.3$ & $95.3 \pm 9.3$ & $103.0 \pm 5.6$ & $107.0 \pm 7.5$ & $119.9 \pm 10.1$ \\
\hline
\end{tabular}

Values are the mean \pm SEM of triplicate tanks. Values in the same line with different superscripts are significantly different $(P<0.05)$

\section{Discussion}

In this study, the effects of the replacement of FO, as the dietary DHA source, with plant oil mixtures containing increasing $\mathrm{AM}$ concentrations on growth performance and fatty acid composition were investigated in juvenile yellowtail. The advantage of high oil productivity/area of microalgae, including Aurantiochytrium sp. (Chisti 2007; Sajjadi et al. 2018), indicates the possibility of AM, as a dietary DHA source, contributing to the sustainability and expansion of aquaculture. The utilization of AM was confirmed in juvenile yellowtail.
The experimental diet was designed to meet the LCPUFA requirement for juvenile yellowtail (approximately $46 \mathrm{~g}$ fish; n-3 LC-PUFA $>2 \%$ in diet) (Deshimaru et al. 1982). Replacement of FO, while meeting the LC-PUFA requirement, did not indicate any significant negative effects on the survival rate of yellowtail (Fukada et al. 2017) and yellowtail king fish (Bowyer et al. 2012). In our study, no significant difference was observed in the survival rate among all dietary groups during the feeding trial. The replacement of FO did not affect fish health during the 8 -week feeding trial. Additionally, daily feed intake did not exhibit any significant differences among the dietary groups. Dietary utilization of AM did not affect feed intake 
in Atlantic salmon (Kousoulaki et al. 2015) and Nile tilapia (Fernandes et al. 2018), but decreased in juvenile tilapia (Sarker et al. 2016) and juvenile olive flounder (Qiao et al. 2014). Thus, AM may have a negative effect on feed intake in some species of juvenile fish.

Although our experimental diets contained enough essential fatty acids for yellowtail, differences were observed in the growth parameters (body weight, weight gain, SGR, and feed efficiency) and liver composition. Furthermore, all experimental diets were prepared with almost the same level of fat; only fatty acid composition differed. The highest growth performance was observed in the AM2 group, which was significantly higher than that of the AM0 group. The high protein efficiency ratio observed in the AM2 group suggests that the dietary lipid was well utilized in fish, known as 'protein sparing effects' (Watanabe 2002). Although the AM0 diet met the n-3 LC-PUFA requirement $(2.18 \%$ in diet), increased dietary inclusion levels of up to $2 \% \mathrm{AM}$ supplementation (2.88\% n-3 LC-PUFA in diet) improved growth performance. The juvenile yellowtails used in the present study were smaller than those used by Deshimaru et al. (1982); therefore, the fish might require higher dietary n-3 LC-PUFA levels at younger stages. In the red sea bream Pagrus major, increasing dietary DHA and/or EPA levels higher than the required levels improved growth and feed efficiency (Takeuchi et al. 1990). Levels of DHA and/or n-3 LC-PUFA higher than the required level might improve growth performance in yellowtail as observed in a previous study (Shinagawa et al. 2017). However, the highest growth performance was observed in the AM2 group that did not contain the highest DHA and n-3 LC-PUFA levels. The optimal n-3 LC-PUFA for SGR was 3.08\%; however, the SGR decreased with higher n-3 LC-PUFA levels (>3.08\%). In tongue sole Cynoglossus semilaevis, the dietary DHA/ EPA ratio affected fish growth and lipid content (Xu et al. 2018). Dietary fatty acid composition might also affect crude protein and lipid levels of the liver, which might be associated with differential body growth. The retention of protein and lipids did not show significant differences; however, the group with higher growth had higher lipid retention. Lipid retention might be associated with better growth in juvenile yellowtail, as previously observed in yellowtail kingfish (Bowyer et al. 2012). The liver is the most important tissue for $\beta$-oxidation in fish (Henderson and Tocher 1987). $\beta$-Oxidation capacities in rat livers increased in response to dietary EPA and DHA (Totland et al. 2000). The difference in dietary fatty acid might change lipid metabolism, followed by body growth. Meeting the essential fatty acid requirements of yellowtail is important; furthermore, a proper balance of dietary fatty acids, including DHA and/or n-3 LCPUFA levels, might also be important.

In the present study, dietary fatty acid composition in the whole body was relatively consistent with that described in a review paper (Turchini et al. 2009). However, the palmitic acid levels in the whole body of the AM1-AM4 groups were low in comparison to that in each diet. Additionally, the levels in the AM1-AM4 groups (21.9-24.8\%) converged with those of the AM0 group (22.4\%), despite the fact that the palmitic acid levels in each diet varied (23.1-34.3\%). This was reflected in the retention of palmitic acid in the AM0-AM4 groups. The decrease and low retention of palmitic acids suggest that the fatty acid might be utilized as an energy source and/or converted to other fatty acids. In fish fed the AM1-AM4 diets, palmitic acid was probably used as an energy source. The EPA levels in the whole body of all dietary groups was lower than that in each diet. Although dietary EPA levels were low in the AM0-AM4 diets, the retention of EPA showed no significant differences among the dietary groups. EPA was probably used as a precursor of DHA and/or for $\beta$-oxidation (Tonial et al. 2009). A certain amount of EPA was stored with any dietary EPA level. The DHA levels in the whole body of all dietary groups was higher than that in each diet. DHA was deposited selectively in the fish body similar to that shown in the results of previous studies (Seno-o et al. 2008; Bowyer et al. 2012). Dietary DHA levels varied in diets; however, the retention of DHA was similar among all dietary groups, including the FO group, in the present study. In tilapia (Brignol et al. 2018), DHA retention was quite high, but decreased slightly together with increasing dietary DHA level. In the orangespotted grouper, DHA retention differed depending on the processing method of Aurantiochytrium sp.; powdered Aurantiochytrium sp. meal showed lower retention than Aurantiochytrium sp. oil and FO (Lee et al. 2017). We used Aurantiochytrium sp. meal produced by the same company as that used in the tilapia study (Brignol et al. 2018), which was different from that used in the study on orange-spotted grouper (Lee et al. 2017). AM used in the present study was the equivalent of FO; therefore, the processing method might be important for its utilization in fish diets.

Differences in oleic acid between the diet and the whole body varied. With regard to levels in the whole body, the AM2 and AM3 groups showed small differences in comparison to the dietary level. Both groups showed high growth. Retention of oleic acid was high in the FO and AM4 groups, whereas the AM2 group had the lowest retention. Oleic acid might be utilized as an energy source. Relatively high levels of linoleic acid were present in the AM0-AM4 groups. Only a small difference was observed in the level between the diet and the whole body; FO and AM2 groups showed high retention, whereas low retention was observed in the AM0 and AM1 groups. Linoleic acid was deposited as fat in the fish body. These data suggest that different fatty acids are utilized differently in yellowtail.

Liver fatty acid composition also reflected the dietary fatty acid composition. MUFA and EPA were low in all 
dietary groups, being used as an energy source or converted to other fatty acids. DHA was higher in the liver of all dietary groups than in each diet, which indicated selective deposition of DHA in the liver. The DHA level in the liver of fish in the present study (approximately 30\%) was high in comparison to our previous studies for yellowtail: $18.8 \%$ in control (Shinagawa et al. 2017) and 8.5\% in control (Fukada et al. 2017). The high levels of DHA observed in the liver could be attributed to the smaller size of the fish in this study compared to those used in previous studies.

For the serum components, significant differences were only observed in total protein concentration. The low-growth group (AM0) exhibited high total protein concentrations in this study compared to a previous study of yellowtail fish, where high total protein concentration was observed in the high-growth group (Fukada et al. 2017). The relationship between total protein concentration and growth is unclear, and thus further investigation is required. When FO was replaced with plant oil, an increase in triglyceride concentration was observed in previous studies of yellowtail (Seno-o et al. 2008; Fukada et al. 2017); however, the triglyceride concentration did not increase in this study. EPA and DHA affect hepatic triglyceride metabolism in mammals, and EPA decreases plasma lipid concentration in rats (Frøyland et al. 1996, 1997). Sufficient dietary n-3 LC-PUFA levels might have contributed to the utilization of triglycerides in the present study.

Replacement of dietary FO using Aurantiochytrium sp. meal (ALL-G RICH ${ }^{\mathrm{TM}}$ ) caused no negative effects on the growth performance and utilization of fatty acids in juvenile yellowtail. A non-FO diet for yellowtail is now available using AM as the dietary DHA source, even though some essential fatty acids are present in FM. Reducing FM as well as FO should be investigated in the future. Furthermore, it is not clear whether juvenile yellowtail require both EPA and DHA or only DHA as essential fatty acids. Therefore, detailed fatty acid requirements for juvenile yellowtail must be determined because AM can only provide DHA as the n-3 LC-PUFA. The best growth performance was observed in the AM2 group; therefore, juvenile yellowtail (initial mean body weight $=19 \mathrm{~g}$ ) might require a high dietary $\mathrm{n}-3$ LC-PUFA level (2.89\%). However, further increase of $n-3$ LC-PUFA did not result in an improvement in fish growth, which suggests that an optimal fatty acid composition in the diet exists for juvenile yellowtail. In the present study, fatty acids from AM were utilized well and retained in juvenile yellowtail. AM is very useful as a DHA source for yellowtail aquaculture and could contribute to reducing the required dietary $\mathrm{FO}$ level and creating a diet without FO. However, algal DHA-rich oil still has a high production cost and a higher price than FO owing to the difficulties of industrial-scale commercial production (Qu et al. 2013; Ryu et al. 2013). Many studies have been performed to reduce production costs (Park et al. 2018; Song et al. 2015), which is expected to help reduce dietary FO levels and contribute to the sustainability and expansion of aquaculture.

Acknowledgements This study was funded by Alltech (USA and Japan) through a joint research alliance between Alltech and Kochi University, Japan.

Open Access This article is licensed under a Creative Commons Attribution 4.0 International License, which permits use, sharing, adaptation, distribution and reproduction in any medium or format, as long as you give appropriate credit to the original author(s) and the source, provide a link to the Creative Commons licence, and indicate if changes were made. The images or other third party material in this article are included in the article's Creative Commons licence, unless indicated otherwise in a credit line to the material. If material is not included in the article's Creative Commons licence and your intended use is not permitted by statutory regulation or exceeds the permitted use, you will need to obtain permission directly from the copyright holder. To view a copy of this licence, visit http://creativecommons.org/licenses/by/4.0/.

\section{References}

Aoki H, Sanada Y, Furuishi M, Kimoto R, Maita M, Akimoto A, Yamagata Y, Watanabe T (2000) Partial or complete replacement of fish meal by alternate protein sources in diets for yellowtail and red sea bream. Aquacult Sci 48:53-63

Bowyer JN, Qin JG, Smullen RP, Srone DAJ (2012) Replacement of fish oil by poultry oil and canola oil in yellowtail kingfish (Seriola lalandi) at optimal and suboptimal temperatures. Aquaculture 356-357:211-222

Brignol FD, Fernandes VAG, Nobrega RO, Corrêa CF, Filler K, Pettigrew J, Fracalossi DM (2018) Aurantiochytrium sp. meal as DHA source in Nile tilapia diet, part II: Body fatty acid retention and muscle fatty acid profile. Aquacult Res 50:707-716

Byreddy AR (2016) Thraustochytrids as an alternative source of omega-3 fatty acids, carotenoids and enzymes. Lipid Tech 28:68-70

Chisti Y (2007) Biodiesel from microalgae. Biotechnol Adv 25:294-306

Deshimaru O, Kuroki K, Yone Y (1982) Suitable levels of lipids and ursodesoxycholic acid in diet for yellowtail. Nippon Suisan Gakkaishi 48:1265-1270

Fernandes VAG, Brignol FD, Filler K, Pettigrew J, Fracalossi DM (2018) Aurantiochytrium sp. meal as DHA source in Nile tilapia diet, part I: Growth performance and body composition. Aquacult Res 50:390-399

Folch J, Lees M, Stanley SGH (1957) A simple method for the isolation and purification of total lipides from animal tissues. J Biol Chem 226:497-509

Frøyland L, Vaagenes H, Asiedu DK, Garras A, Lie Ø, Berge RK (1996) Chronic administration of eicosapentaenoic acid and docosahexaenoic acid as ethyl esters reduced plasma cholesterol and changed the fatty acid composition in rat blood and organs. Lipids 31:169-178

Frøyland L, Madsen L, Vaagenes H, Totland GK, Auwerx J, Kryvi H, Staels B, Berge RK (1997) Mitochondrion is the principal target for nutritional and pharmacological control of triglyceride metabolism. J Lipid Res 38:1851-1858

Fukada H, Taniguchi E, Morioka K, Masumoto T (2017) Effects of replacing fish oil with canola oil on the growth performance, fatty acid composition and metabolic enzyme activity of juvenile 
yellowtail Seriola quinqueradiata (Temminck \& Schlegel, 1845). Aquacult Res 48:5928-5939

García-Ortega A, Kissinger KR, Trushenski JT (2016) Evaluation of fish meal and fish oil replacement by soybean protein and algal meal from Schizochytrium limacinum in diets for giant grouper Epinephelus lanceolatus. Aquaculture 452:1-8

Hardy RW (2010) Utilization of plant proteins in fish diets: effects of global demand and supplies of fishmeal. Aquacult Res 41:770-776

Henderson RJ, Sargent JR (1985) In Nutrition and Feeding in Fish (CB Cowey, AM Mackie and JG Bell, Eds) Fatty acid metabolism in fish: $349-364$

Henderson RJ, Tocher DR (1987) The lipid composition and biochemistry of freshwater fish. Prog Lipid Res 26:281-347

Kissinger KR, García-Ortega A, Trushenski JT (2016) Partial fish meal replacement by soy protein concentrate, squid and algal meals in low fish-oil diets containing Schizochytrium limacinum for longfin yellowtail Seriola rivoliana. Aquaculture 452:37-44

Kousoulaki K, Østbye T-KK, Krasnov A, Torgersen JS, Mørkøre T, Sweetman J (2015) Metabolism, health and fillet nutritional quality in Atlantic salmon (Salmo salar) fed diets containing n-3-rich microalgae. J Nutr Sci 4:e24

Lee MC, Zhuo LC, Lin Y-H (2017) Effects of dietary docosahexaenoic acid sources, microalgae meal and oil, on growth, fatty acid composition and docosahexaenoic acid retention of orange-spotted grouper, Epinephelus coioides. Aquacult Res 49:30-35

Masumoto T (2002) Yellowtail, Seriola quinqueradiata (CD Webster and CE Lim, Eds.). Nutrient requirements and feeding of finfish for Aquaculture:131-146

Masumoto T, Ruchimat T, Ito Y, Hosokawa H, Shimeno S (1996) Amino acid availability values for several protein sources for yellowtail (Seriola quinqueradiata). Aquaculture 146:109-119

Miller MR, Nichols PD, Carter CG (2007) Replacement of fish oil with thraustochytrid Schizochytrium sp. L oil in Atlantic salmon parr (Salmo salar L) diets. Comp Biochem Physiol A 148:382-392

Park W, Moon M, Shina S, Cho JM, Suh WI, Chang YK, Lee B (2018) Economical DHA (Docosahexaenoic acid) production from Aurantiochytrium sp. KRS101 using orange peel extract and low cost nitrogen sources. Algal Res 29:71-79

Qiao H, Wang H, Song Z, Ma J, Li B, Liu X, Zhang S, Wang J, Zhang L (2014) Effects of dietary fish oil replacement by microalgae raw materials on growth performance, body composition and fatty acid profile of juvenile olive flounder, Paralichthys olivaceus. Aquacult Nutr 20:646-653

Qu L, Ren LJ, Huang H (2013) Scale-up of docosahexaenoic acid production in fed-batch fermentation by Schizochytrium sp. based on volumetric oxygen-transfer coefficient. Biochem Eng J 77:82-87

Ratledge C (2004) Fatty acid biosynthesis in microorganisms being used for single cell oil production. Biochimie 86:807-815

Ryu BG, Kim K, Kim J, Han JI, Yang JW (2013) Use of organic waste from the brewery industry for high-density cultivation of the docosahexaenoic acid-rich microalga, Aurantiochytrium sp. KRS101. Biores Technol 129:351-359

Sajjadi B, Chen WY, Raman AAA, Ibrahim S (2018) Microalgae lipid and biomass for biofuel production: A comprehensive review on lipid enhancement strategies and their effects on fatty acid composition. Renew Sustain Energy Rev 97:200-232

Sargent JR, Tocher DR, Bell JG (2002) The lipids. In: Halver JE, Hardy RW (eds) Fish nutrition, 3rd edn. New York, pp 181-257

Sarker PK, Gamble MM, Kelson S, Kapuscinski AR (2015) Nile tilapia (Oreochromis niloticus) show high digestibility of lipid and fatty acids from marine Schizochytrium sp. and of protein and essential amino acids from freshwater Spirulina sp. feed ingredients. Aquacult Nutr 22:109-119

Sarker PK, Kapuscinski AR, Lanois AJ, Livesey ED, Bernhard KP, Coley ML (2016) Towards sustainable aquafeeds: complete substitution of fish oil with marine microalga Schizochytrium sp. improves growth and fatty acid deposition in juvenile Nile tilapia (Oreochromis niloticus). (JL Soengas, Ed.). PLoS One 11:e0156684

Seno-o A, Takakuwa F, Hashiguchi T, Morioka K, Masumoto T, Fukada H (2008) Replacement of dietary fish oil with olive oil in young yellowtail Seriola quinqueradiata: effects on growth, muscular fatty acid composition and prevention of dark muscle discoloration during refrigerated storage. Fish Sci 74:1297-1306

Shinagawa J, Morino H, Masumoto T, Fukada H (2017) Development of a docosahexaenoic acid (DHA)-rich yellowtail Seriola quinqueradiata using tuna by-product oil. Fish Sci 83:607-617

Song X, Zhang X, Zhang X (2015) Production of High Docosahexaenoic acid by Schizochytrium sp. using low-cost raw materials from food industry. J Oleo Sci 64:197-204

Sprague M, Walton J, Campbell PJ, Strachan F, Dick JR, Bell JG (2015) Replacement of fish oil with a DHA-rich algal meal derived from Schizochytrium sp. on the fatty acid and persistent organic pollutant levels in diets and flesh of Atlantic salmon (Salmo salar, L.) post-smolts. Food Chem 185:413-421

Takeda M, Shimeno S, Hosokawa H, Amano K, Ikeda K, Inoue I (1989) Effects of supplemental dietary oxidized oil and nutrient mixture on lipid peroxidation in red sea bream. Suisanzousyoku $37: 1-7$

Takeuchi T, Toyota M, Satoh S, Watanabe T (1990) Requirement of juvenile red seabream Pagrus major for eicosapentaenoic and docosahexaenoic acids. Nippon Suisan Gakkaishi 56:1263-1269

Tocher DR (2010) Fatty acid requirements in ontogeny of marine and freshwater fish. Aquacult Res 41:717-732

Tonial IB, Stevanato FB, Matsushita M, De Souza NE, Furuya WM, Visentainer JV (2009) Optimization of flaxseed oil feeding time length in adult Nile tilapia (Oreochromis niloticus) as a function of muscle omega-3 fatty acids composition. Aquacult Nutr 15:564-568

Totland GK, Madsen L, Klemetsen B, Vaagenes H, Kryvi H, Frøyland L, Hexeberg S, Berge RK (2000) Proliferation of mitochondria and gene expression of carnitine palmitoyl transferase and fatty acyl-CoA oxidase in rat skeletal muscle, heart and liver by hypolipidemic fatty acids. Biol Cell 92:1-13

Turchini GM, Torstensen BE, Ng WK (2009) Fish oil replacement in finfish nutrition. Rev Aquacult 1:10-57

Watanabe T (2002) Strategies for further development of aquatic feeds. Strategies for further development of aquatic feeds. Fish Sci 68:242-252

Xu H, Cao L, Wei Y, Zhang Y, Liang M (2018) Lipid contents in farmed fish are influenced by dietary DHA/EPA ratio: a study with the marine flatfish, tongue sole (Cynoglossus semilaevis). Aquaculture 485:183-190

Publisher's Note Springer Nature remains neutral with regard to jurisdictional claims in published maps and institutional affiliations. 